Rev. Elev. Méd. vét. Pays trop., 1975, 28 (3) : 319-329.

\title{
Observations complémentaires sur les lépidoptères ophtalmotropes en Afrique occidentale
}

\author{
par W. BUTTIKER $\left({ }^{*}\right)$ et J. NICOLET $\left({ }^{* *}\right)$
}

\begin{abstract}
RESUME
Une deuxième expédition au sujet des lépidoptères ophtalmotropes a eu lieu en 1972 en Côte d'Ivoire et en Haute-Volta dans le but de mener des recherches complémentaires d'ordre entomologique et microbiologique. Papillons ophtalmotropes considérés: les noctuidés Arcyophora patricula Hpsn., A. longivalvis Guen., $A$. zanderi Feld. et $A$. endoglauca Hpsn. Au cours d'une excursion spéciale en Haute-Volta, on a trouvé Arcyophora patricula aux alentours de Bobo-Dioulasso. Nous avons pu confirmer les mours ophtalmotropes chez les espèces : Sylepta derogata Fabr. (Pyralidae) et Scopula lubricata Warr. (Geometridae).

Cependant, aucun exemplaire des sphingidés suspects, Nephele comma (Hopfer) et $N$. peneus (Cramer) n'a été observé pendant ces dernières excursions dans les régions de Korhogo, Bouaké, Abokouamékro et Abidjan. On a fait des observations supplémentaires sur les biotopes; les résultats obtenus au sujet de la transmission de la kératoconjonctivite épidémique aux bovins sont publiés séparément. Au stade larvaire, Mermis sp., un ver endoparasite, a été isolé d'une femelle d'Arcyophora patricula (près de Bouaké).
\end{abstract}

\section{INTRODUCTION}

Différentes espèces de lépidoptères ophtalmotropes ont été déterminées en Afrique occidentale sur les bovins et sur le cheval en Guinée (13), au Nigéria (3), en Côte d'Ivoire (6), au Mali (7). On connaît, dans d'autres pays d'Afrique occidentale, des papillons manifestant ces mours alimentaires particulières avec un hôte mammifère. Une première expédition entomologique s'est déroulée à ce sujet en Côte d'Ivoire en 1970, dont les résultats furent publiés récemment (6) avec une bibliographie très complète au point de vue historique, répartition, biologie, éventail des hôtes et aspects éco-

Travail réalisé grâce à l'appui financier du Fonds National Suisse de la Recherche Scientifique, requête n० 3.503.71.

(*) Ciba-Geigy S.A., CH-4002 Bâle, Suisse.

(**) Institut bactériologique Université, CH-3001 Berne, Suisse. logiques de ces papillons extraordinaires d'Afrique, d'Asie et d'Amérique du Sud. On a reçu depuis lors des informations supplémentaires en matière d'écologie végétale de la Côte d'Ivoire, informations utilisées dans cette publication. C'est surtout la publication de GUILLAUMET et ADJANOHOUN (12) qui a permis des rectifications en ce qui concerne le système phytoécologique dans la région de Korhogo et aux confins des savanes guinéenne et soudanaise.

Nous avons exposé dans la présente publication les observations entomologiques faites pendant la deuxième expédition en Afrique occidentale, en établissant des comparaisons avec les résultats obtenus pendant l'expédition préliminaire de 1970.

En ce qui concerne les recherches microbiologiques menées en Côte d'Ivoire, elles sont résumées dans une publication précédente (15). 


\section{RESULTATS ENTOMOLOGIQUES}

25 excursions nocturnes en Côte d'Ivoire et une excursion en Haute-Volta. On a recueilli au total 157 exemplaires de lépidoptères ophtalmotropes. Pendant la période des travaux en plein champ, 37 troupeaux de bovins furent visités dans 18 villages ou autres localités.

Durant les mois d'octobre et de novembre 1972 , on a prélevé les espèces et exemplaires suivants : Noctuidae (Westermanniinae);

\section{- Arcyophora patricula Hampson 1902}

Cette espèce est la plus commune des lépidoptères ophtalmotropes de Côte d'Ivoire. Les prélèvements de l'expédition 1972 ont corroboré la situation signalée en 1970.

Elle est répartie assez généralement dans les savanes d'Afrique occidentale, orientale et centrale. C'est surtout dans le secteur soudanais que cette espèce est nombreuse.

Les exemplaires recueillis en Haute Volta constituent la première observation dans ce pays (tableau $\mathbf{n}^{\circ} \mathrm{I}$ ).

Grâce à l'aimable soutien de M. Renwart, d'Abokouamékro, nous avons reçu un mâle et quatre femelles supplémentaires de $A$. patricula, prélevés le 4 juillet 1970 au même endroit sur les yeux de bovins.

\section{- Arcyophora longivalvis Guenée 1852}

Cette espèce a été retrouvée à plusieurs reprises pendant la dernière expédition en Côte d'Ivoire en 1972 (tableau $n^{\circ}$ II). La répartition en Afrique occidentale est indiquée sur la carte (p. 322).

Une comparaison avec les conditions existant en Afrique septentrionale au sujet de l'éventail des espèces a fait l'objet de travaux récents [BUTTTIKER $(6,7)]$. A. longivalvis s'est avéré l'espèce la plus commune en Afrique du sudouest et en Afrique du Sud. Dans les recherches menées en Côte d'Ivoire, $A$. patricula fut l'espèce la plus répandue pendant les expéditions en 1970 et 1972 .

TABL. N ${ }^{\circ}$ I-Prêlēvement de Arcyophora patricuila Hampson 1902 en Côte d'Ivoire et en Haute-Volta.

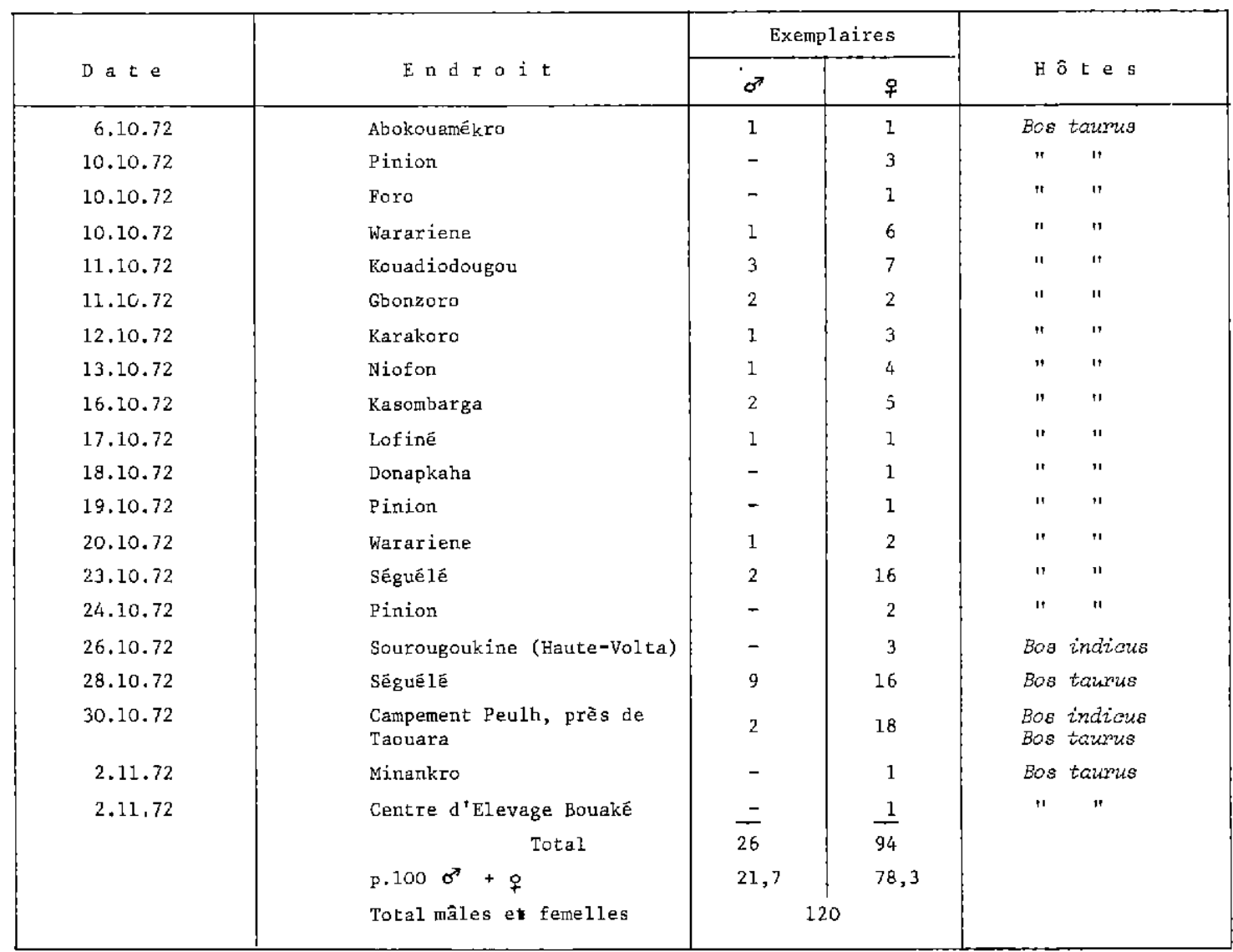


TABL. N ${ }^{\circ}$ II-Prélèvement de Arcyopisora longivalvis Guenể 1852 en Côte d'Ivoire.

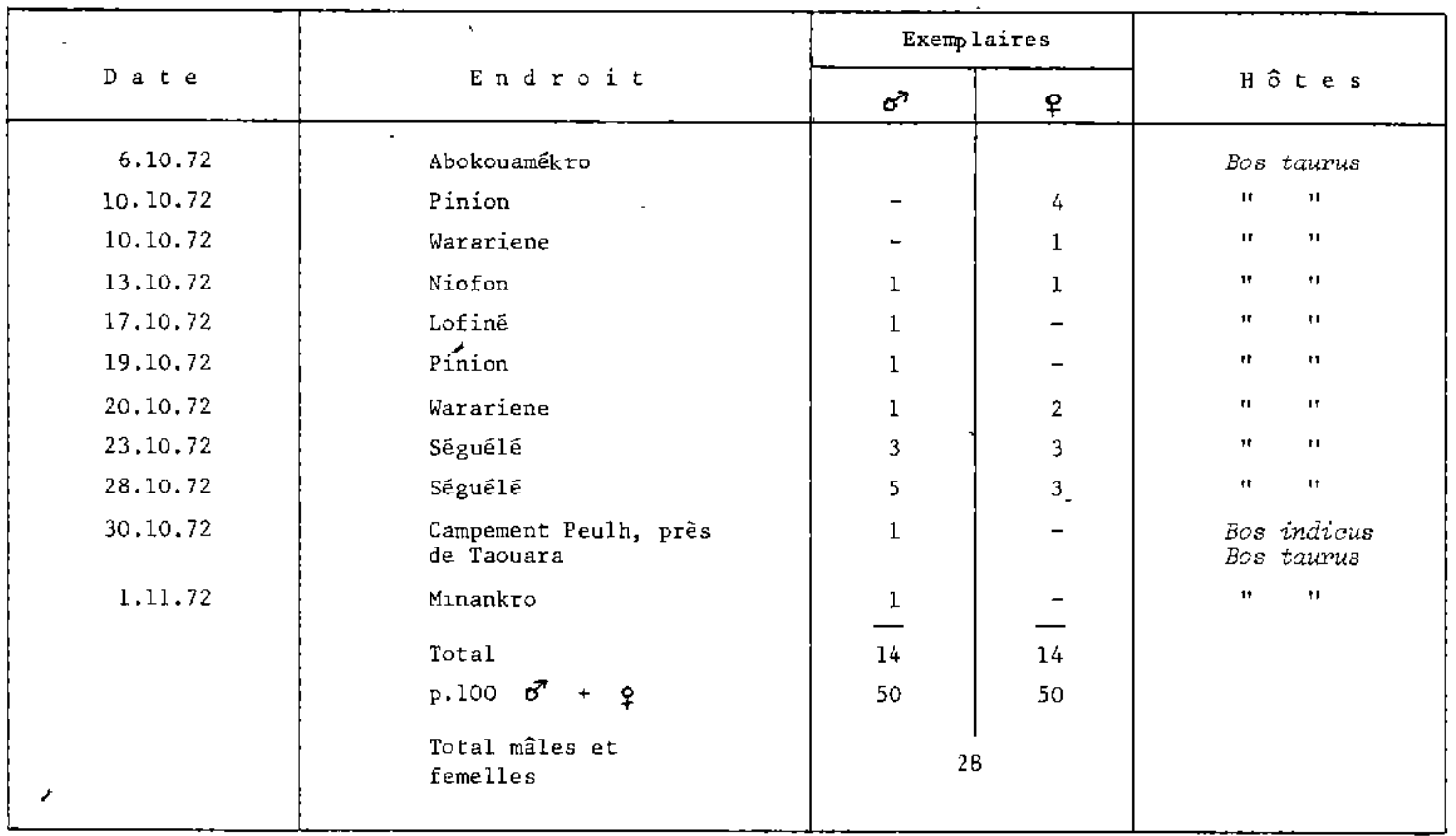

- Arcyophora zanderi Felder 1875

- 10-10-1972, Warariene, 2 O. Hôte : Bos taurus.

- 11-10-1972, Kouadiodougou, 1 ․ Hôte : Bos taurus.
- Arcyophora (Setoctena) endoglauca Hampson 1910

- 30-10-1972, Campement Peuhl, près de Taouara $10^{\prime}+1$. Hôte : Bos indicus.

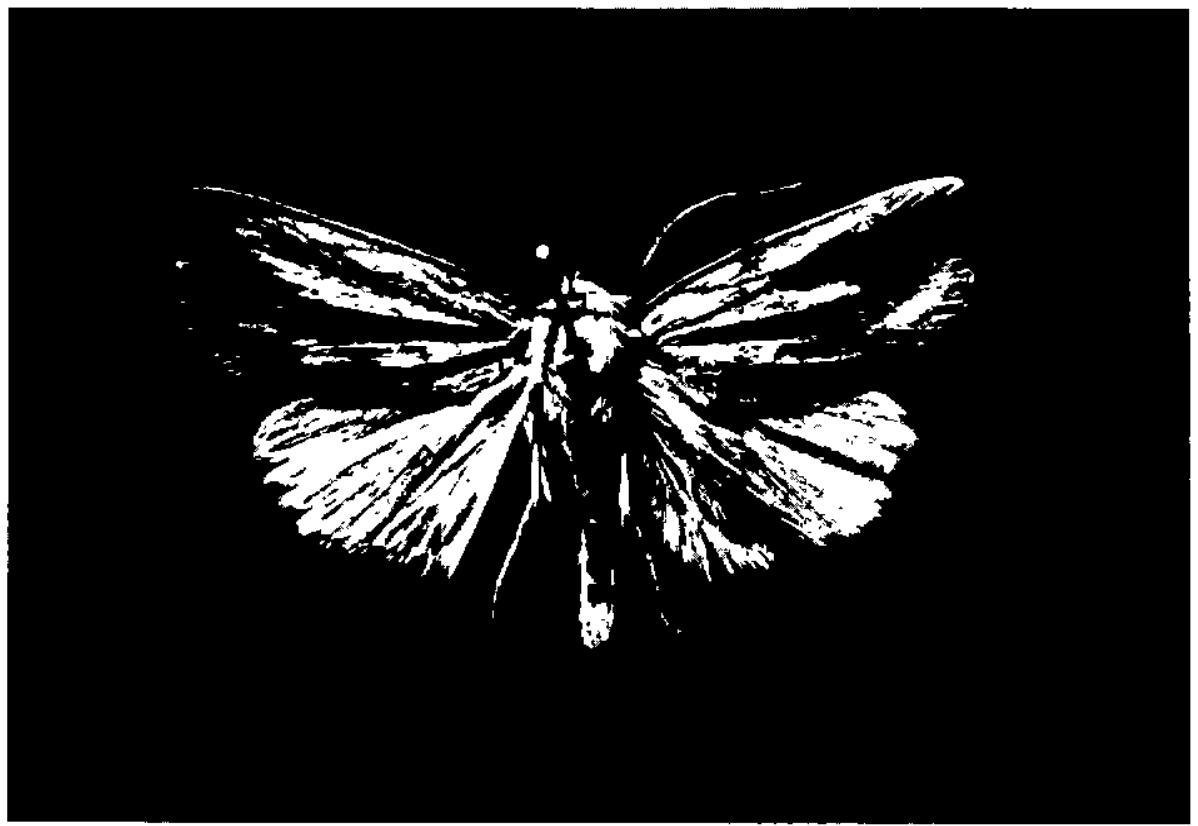

Fig. 1. - Arcyophora endoglauca, femelle, prélevée à Taouara; envergure $20 \mathrm{~mm}$. 


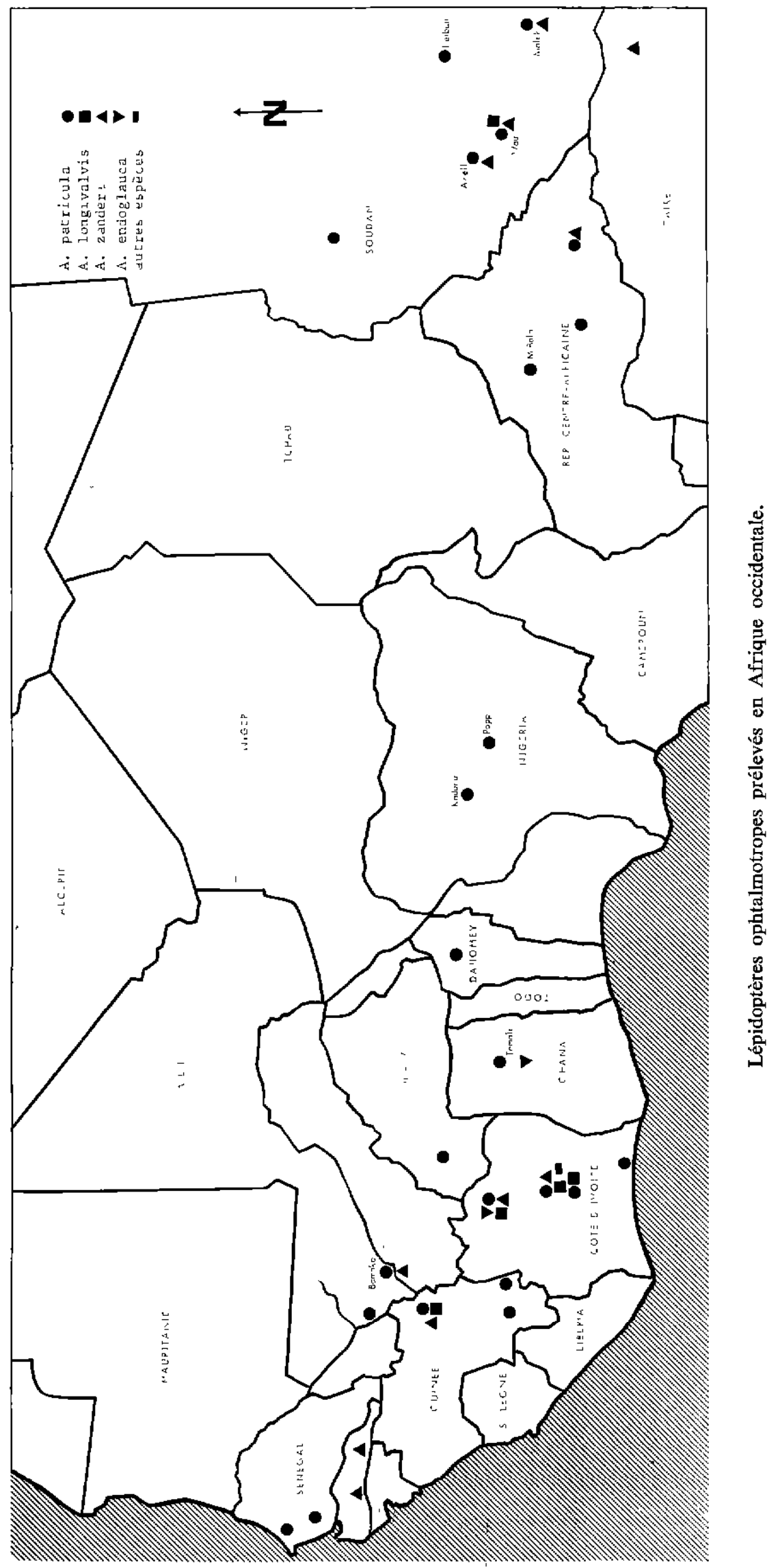


A l'occasion d'une excursion nocturne en Haute-Volta, on a observé un assez grand nombre de noctuidés Arcyophora patricula sur quatre troupeaux de bovins aux alentours de BoboDioulasso. Après un violent orage, trois exemplaires de cette espèce ont été prélevés sur les zébus appartenant aux bergers Peuhl.

Il s'est avéré que pendant la tempête tropicale les papillons étaient absents; cependant, à la fin des précipitations, des individus se manifestaient graduellement, très probablement à partir de la brousse avoisinante.

\section{Pyralidae (Pyraustinae)}

- Sylepta derogata Fabr.

- 16-10-1972, Kasombarga, 1 expl. Hôte : Bos taurus.

- 30-10-1972, Taouara (Campement), 1 expl.

Hôte: Bos taurus.

Les deux exemplaires ont été prélevés des yeux des bovins à deux endroits très proches et où la végétation de la brousse est presque identique.

Quatre autres espèces du genre Sylepta se sont révélées des lépidoptères ophtalmotropes :

- Sylepta sp. près de fulviceps Beth.-Baker, sur bovins en Côte d'Ivoire (6).

- Sylepta leopardalis Moore, occasionnellement en Malaisie, sur le corps de buffles et de sambars, et rarement sur leurs yeux (1).

- Sylepta sp. près de iopardalis Wlk., assez commun en Thaïlande sur un grand nombre d'hôtes (1).

Un exemplaire de Sylepta balteata Fabr, a été prélevé au Nigéria au village de Malali dans les alentours de Kadouna le 30 avril 1970. I s'agit d'une observation fortuite non mentionnée dans la publication concernant les recherches faites au Nigéria (3).

Les mêmes précisions s'appliquent à une autre pyralide Bradina admixtalis Wlk., trouvée à Anyal, près de Kadouna au Nigéria le 24 juin 1971. Pour les deux dernières espèces l'hôte était la vache domestique.

- Geometridae (Sterrhinae)

Scopula lubricata Warr.

- 28-10-1972, Séguélé, 2 exemplaires. Hôte : Bos taurus.

Cette espèce est évidemment très rare.
L'exemplaire trouvé à Piniono pendant l'expédition de 1970 nous semble identique à l'exemplaire de Séguélé.

En Asie, trois espèces de Scopula ont été observées comme ophtalmotropes $(2,1)$ : Scopula attentata, $S$. pulverosa et $S$. fibulata.

Les observations faites en 1970 et 1972 sont comparées ci-après. Elles reflètent des conditions similaires au point de vue de la composition effective et relative de l'éventail des espèces et des proportions des sexes, surtout pour le genre Arcyophora (tableaux III et IV). Il est nécessaire de mentionner que les recherches faites étaient concentrées vers des travaux microbiologiques d'où le moindre nombre des prélèvements des papillons. Cependant le nombre des lépidoptères ophtalmotropes était lui aussi moins grand qu'en 1970 (tableau V).

\begin{tabular}{|c|c|c|c|c|}
\hline \multirow[t]{2}{*}{ Espèces } & \multicolumn{2}{|c|}{$\begin{array}{l}1970 \\
\text { Côte d'Ivoire } \\
\text { (Büttiker 1973) }\end{array}$} & \multicolumn{2}{|c|}{$\begin{array}{l}1972 \\
\text { Côte d'Ivoire } \\
\text { et Haute-Volta } \\
\text { (la présente pu- } \\
\text { blication) }\end{array}$} \\
\hline & $\mathrm{n}$ & p. 100 & $\mathrm{n}$ & p.100 \\
\hline A. patricuza & 314 & 86,7 & $120^{+}$ & $76,5^{+}$ \\
\hline A. Zongivazvis & 23 & 6,3 & 28 & 17,8 \\
\hline A. zonderi & 18 & 5,0 & 3 & 2,0 \\
\hline A. endoglauca & 2 & 0,6 & 2 & 1,2 \\
\hline $\begin{array}{l}\text { autres espẽces } \\
\text { ophtalmotropes }\end{array}$ & 5 & 1,4 & 4 & 2,5 \\
\hline Total & 362 & 100,0 & 157 & 100,0 \\
\hline
\end{tabular}

+ y compris les exemplaires de Sourougoukine, (Haute-Volta).

TABL. $N^{\bullet} I V$-Pourcentage de mâles et femelles des différentes espèces du genre Arcyophora.

\begin{tabular}{|c|c|c|c|c|}
\hline \multirow[t]{2}{*}{ Espèces } & \multicolumn{2}{|c|}{$\begin{array}{l}1970 \\
\text { Côte d'Ivoıre } \\
\text { (Büttikes 1973) }\end{array}$} & \multicolumn{2}{|c|}{$\begin{array}{l}972 \\
\text { Côte d'Ivoire et } \\
\text { Haute-Volta } \\
\text { (la presente pu- } \\
\text { blication) }\end{array}$} \\
\hline & $\begin{array}{c}p .100 \\
\sigma\end{array}$ & $\begin{array}{c}\text { p. } 100 \\
q\end{array}$ & $\begin{array}{c}p .100 \\
\sigma^{2}\end{array}$ & $\begin{array}{c}p .100 \\
+\end{array}$ \\
\hline A. patricuta & 11 & 89 & $21.7^{+}$ & $78.3^{+}$ \\
\hline A. Zongivalvis & 30 & 70 & 50.0 & 50.0 \\
\hline A. zandem & 11 & 89 & - & 100.0 \\
\hline 4. endoglauca & 50 & 50 & 50.0 & 50.0 \\
\hline
\end{tabular}

$+y$ compris les exemplaires de Sourougoukine (Haute-Volta). 
TABLEAU $\mathrm{N}^{\circ} \mathrm{V}$

\begin{tabular}{|l|r|r|}
\hline \multicolumn{1}{|c|}{ Critères } & 1970 & 1972 \\
\hline Nombre de bovins présents & 3316 & I 733 \\
Nombre de lépidoptères prélevés & 362 & 157 \\
$\begin{array}{l}\text { Nombre total de lépidoptères } \\
\text { ophtalmotropes observés }\end{array}$ & 2750 & 740 \\
$\begin{array}{l}\text { Nombre de lépidoptêres observés } \\
\text { par bovin (nombre total) }\end{array}$ & 0.83 & 0.43 \\
$\begin{array}{l}\text { Nombre de lépidoptères prēlevês } \\
\text { par bovin (nombre total) }\end{array}$ & 0.11 & 0.08 \\
$\begin{array}{l}\text { Nombre maximal de lépidoptères } \\
\text { ophtalmotropes observés sur un } \\
\text { oeil de 1'hôte }\end{array}$ & 6 & 4 \\
\hline
\end{tabular}

En ce qui concerne l'abondance des lépidoptères ophtalmotropes dans les troupeaux de bovins inspectés, les chiffres indiqués au tableau $\mathrm{V}$ reflètent les observations faites en 1972, comparées à celles de 1970.

\section{Le genre Arcyophora en Afrique occidentale}

En ce qui concerne la répartition du genre Arcyophora dans les pays de l'Afrique occidentale, voir le tableau VI.

Ce tableau reflète la distribution des différentes espèces, les hôtes observés et les conditions phytoécologiques indiquées par les catégories de savanes existantes là où les lépidoptères ont été prélevés.

Par l'intermédiaire de $\mathrm{M}$. M. Bréfin de Parakou (Dahomey), j'ai reçu deux femelles de Arcyophora patricula, prélevées à Banikoara sur un bovin, le 30 mars 1973. Le troupeau comprenait environ 100 têtes et le nombre maximal de lépidoptères observés sur un seul œil fut 5 exemplaires. D'après la description de la végétation du lieu de prélèvement, elle se rattache à la savane à brousse épineuse avec ça et là des arbres d'assez grande taille.

TABL. $\mathrm{N}^{\circ} \mathrm{VI}$-Répartition des espèces du genre Aroyophora, hôtes des adultes et type de savanes.

\begin{tabular}{|c|c|c|c|c|}
\hline Pays & Espèces & Localitês & Hôtes & A u t e u r s \\
\hline Guinęe & $\begin{array}{l}\text { A. zanderi } \\
\text { A. patricula } \\
\text { A. Zongivaivis }\end{array}$ & $\begin{array}{l}\text { Diodougau } \\
\text { Beyla } \\
\text { Forekariah } \\
\text { Oudoula } \\
\text { Siguiri } \\
\text { Dioudougou }\end{array}$ & $\begin{array}{c}\text { Equas acbatitus } \\
- \\
- \\
- \\
- \\
-\end{array}$ & $\begin{array}{l}\text { de Joannis (1911) } \\
\text { Fletcher (1966); } \\
\text { Buttiker (1973 a) } \\
\text { Fletcher (1966) } \\
\text { Fletcher (1966) } \\
\text { Viette (1970) } \\
\text { de Joannis (1911) }\end{array}$ \\
\hline Sënëga1 & $\begin{array}{l}\text { A. aconderi } \\
\text { A. patricuza }\end{array}$ & $\begin{array}{l}\text { Sebdou } \\
\text { Bambey; Kaolack }\end{array}$ & - & $\begin{array}{l}\text { F1etcher }(1966) \\
\text { F1etcher }(1966)\end{array}$ \\
\hline Gambie & A. zonderi & Casamance & - & Fletcher (1966) \\
\hline Mali & $\begin{array}{l}\text { A. zanderei } \\
\text { A. patricuta }\end{array}$ & $\begin{array}{l}\text { Bamako } \\
\text { Bamako } \\
\text { Bamako }\end{array}$ & $\begin{array}{c}- \\
- \\
\text { Bos taurus }\end{array}$ & $\begin{array}{l}\text { Viette }(1970) \\
\text { Viette }(1970) \\
\text { Büttiker }(1973 \mathrm{~b})\end{array}$ \\
\hline $\begin{array}{l}\text { Côte } \\
\text { d'Ivoire }\end{array}$ & $\begin{array}{l}\text { A. patriauta } \\
\text { A. iongivaivis } \\
\text { A. zanderi } \\
\text { A. endogiauca }\end{array}$ & $\begin{array}{l}\text { dìverses régions ) } \\
\text { divers endrouts ) } \\
\text { ) }\end{array}$ & $\begin{array}{l}\text { Bos tourus } \\
\text { Bos indious } \\
\text { Eques cabatius }\end{array}$ & $\begin{array}{l}\text { Büttiker (1973 a) } \\
\text { et cette publication) }\end{array}$ \\
\hline Ghana & $\begin{array}{l}\text { A. patricuta } \\
\text { A. endogiausa }\end{array}$ & $\begin{array}{l}\text { Kete Krachi; Tamale; } \\
\text { Gambaga } \\
\text { Kete Krachi }\end{array}$ & $\begin{array}{l}- \\
-\end{array}$ & $\begin{array}{l}\text { Fletcher (1966) } \\
\text { Fletcher (1966) }\end{array}$ \\
\hline Nigeria & A. patricuia & $\begin{array}{l}\text { Kaduna } \\
\text { Ropp }\end{array}$ & $\begin{array}{c}\text { Bos indiaus } \\
-\end{array}$ & $\begin{array}{l}\text { Büttıker (1970) } \\
\text { Fletcher (1966) }\end{array}$ \\
\hline R.C.A. & $\begin{array}{l}\text { A. patriauta } \\
\text { A. zanderi }\end{array}$ & $\begin{array}{l}\text { Koulouba } \\
\text { Koulouba }\end{array}$ & - & $\begin{array}{l}\text { viette }(1970) \\
\text { viette }(1970)\end{array}$ \\
\hline Haute-Volta & $\begin{array}{l}\text { A. aconderi } \\
\text { A. patricuta }\end{array}$ & $\begin{array}{l}\text { Dioudougou } \\
\text { Sourougoukine } \\
\text { (Bobo-Dioulasso) }\end{array}$ & $\begin{array}{c}- \\
\text { Bos unäious }\end{array}$ & $\begin{array}{l}\text { Fletoher (1966) } \\
\text { Büttiker (cette publication) }\end{array}$ \\
\hline Dahomey & A. patrioula & Banjkoara & Bos taurus & - \\
\hline
\end{tabular}


Ces exemplaires de Banikoara sont les premiers lépidoptères ophtalmotropes, prélevés au Dahomey.

Des recherches supplémentaires ont été faites au sujet des diptères hôtes de bovins domestiques. D'une manière très générale, on a observé qu'un grand nombre de Muscidae étaient présents sur la tête et autour des yeux des bovins. Les espèces collectionnées, obligeamment identifiées par M. A. C. PONT, Londres, sont les suivantes: Stomoxys nigra Macquart, Musca fasciata Stein, $M$. alpesa Walker, $M$. domestica Linnaeus, $M$. tempestiva Bezzi, $M$. xanthomelas Wiedemann, $M$. lusoria Wiedemann,

Les différentes espèces de Muscidae se nourrissent de sang et des sécrétions des orifices de la tête; souvent ces diptères sont particulièrement attirés par les yeux. D'après PONT (1973), on pourrait s'attendre à observer Musca sorbens, une espèce très pestilentielle qui fréquente les yeux des mammifères. Il est probable que, pendant les périodes d'expédition, le temps était trop sec pour cela.

\section{PARASITES ET ENNEMIS}

Un exemplaire d'un mermithide, au stade larvaire, a été trouvé dans le haemocoel d'une femelle Arcyophora patricula prélevée à Minankro près de Bouaké, le 11 novembre 1973. Le papillon a été disséqué pour les travaux microbiologiques; l'insecte ne manifesterait pas d'anomalies externes. Malheureusement, l'identification spécifique de ce parasite ne fut pas possible vu son stade trop jeune. Il faut rappeler qu'en Thailande le papillon ophtalmotrope, Lobocraspis griseifusa Hpsn., s'est avéré un hôte de Mermis sp. (2).

Les mermithides sont connus comme parasites d'au moins 15 ordres d'insectes. Selon l'espèce de l'hôte et du parasite, l'insecte est infesté par pénétration active de la larve à travers la cuticule ou, moins fréquemment, par ingestion de la larve ou de l'œuf du parasite par l'hôte (17). La plupart des 300 observations faites sur les mermithides comme parasites des insectes se réfère aux insectes médicaux [Chironomidae, Culicidae et Simulidae (21)]. En Afrique et Amérique centrale, le genre Simulium est assez souvent parasité par les mermithides. En ce qui concerne la mor- talité provoquée par les mermithides chez les simulies, les pourcentages varient de moins de 1 à 100 p. 100 .

A plusieurs reprises, nous avons remarqué pendant les excursions nocturnes que des chauves-souris (dont l'identification ne fut malheureusement pas possible) se sont approchées à petite distance du bétail, attrapant des papillons en volant assez lentement parmi les troupeaux de bovins des kraals. C'est surtout à Foro, Séguélé, Karakoro, Piniono et Niofon que les chauves-souris étaient les plus nombreuses et les plus actives. Nous avons eu l'impression qu'un grand nombre de papillons ont été $a b-$ sorbés par ces chiroptères.

\section{HOTES}

Les recherches faites lors de cette dernière expédition furent concentrées sur du bétail domestique. Cependant, on a saisi l'occasion de faire des observations sur des hôtes potentiels ou supplémentaires. Au total, les recherches s'étendirent aux espèces suivantes : bovins, caprins, ovins, porcins, équins, chiens et au point de vue animaux sauvages au Cephalophus (Philantomba maxwelli).

A l'exception des bovins chez qui le papilIon a été observé de façon plus fréquente chez Bos taurus que chez Bos indicus, aucune des autres espèces n'a été observée servant d'hôte.

Malgré des efforts considérables, nous n'avons pas pu trouver des lépidoptères ophtalmotropes sur les mammifères autres que les bovins.

\section{ECOLOGIE}

Les observations de la dernière expédition (1972) ont généralement confirmé les résultats obtenus en 1970. Il s'est de nouveau avéré que la répartition des lépidoptères ophtalmotropes est assez prononcée dans la savane guinéenne et soudanaise.

En comparant les travaux écologiques des auteurs cités dans le rapport de l'expédition 1970 et la récente publication de GUILLAUMET et ADJANOHOUN (12), on constate que la limite méridionale de la savane guinéosoudanaise est située plus au sud que ce que 
I'on avait indiqué dans les publications précédentes. Cette nouvelle situation nécessite une redélimitation de la frontière entre les savanes en question. La chose a été faite dans la nouvelle carte écologique. Les localités des prélèvements récents en Haute-Volta et au Dahomey se trouvent également dans la zone soudanaise.

Les informations plus récentes obtenues au cours des différentes expéditions confirment la situation décrite dans le travail de 1970. Des informations supplémentaires en provenance des pays du Sahel montrent également que la distribution de ces papillons particuliers est plus rare dans la savane sahélienne. Les conditions particulières à cette région nécessitent des recherches plus approfondies.

Rappelons que différentes espèces des genres Combretum et Terminalia sont plus ou moins régulièrement réparties dans les savanes d'Afrique et les forêts à feuilles caduques en
Asie. Il nous semble justifié de choisir ces deux genres comme lignes indicatrices de la répartition des lépidoptères ophtalmotropes, particulièrement du genre Arcyophora.

Au point de vue du bioclimat, les faits suivants sont importants car le régime des pluies, la totalité des précipitations, la durée de la saison sèche et le déficit de l'humidité exercent une influence considérable sur les types de végétation et la faune tributaire de cette végétation. Ces aspects ont fait l'objet d'investigations approfondies dans une publication précédente (6).

\section{CONCLUSIONS}

Les observations faites en 1970 ont été largement corroborées par celles de la dernière expédition en Côte d'Ivoire soit, en ce qui

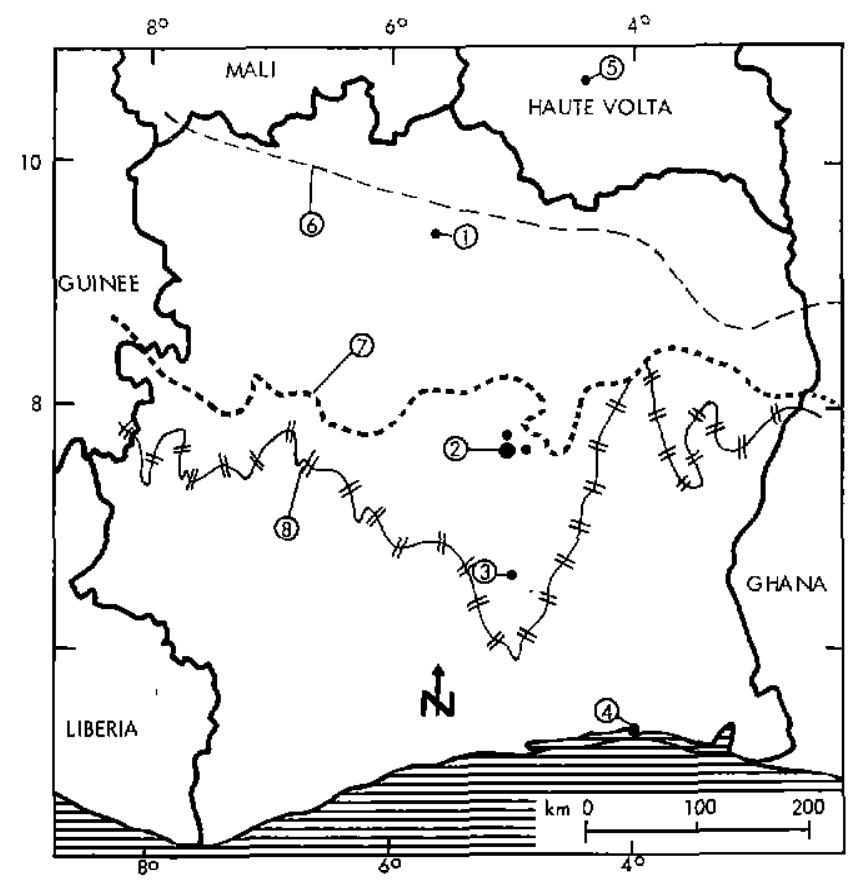

Carte écologique (d'après Guillaumet et Adjanohoun, 1971). Limites et secteurs des domaines guinéens et soudanais et de la forêt dense. Endroits ou districts des prélèvements.

1. Korhogo.

2. Bouaké.

3. Abokouamékro.

4. Adiopodoumé/Abidjan.

5. Bobo-Dioulasso.

6. Limite des secteurs soudanais (nord) et sub-soudanais (sud).

7. Limite du secteur sub-soudanais (nord) et savane guinéenne (sud).

8. Limite de la savane guinéenne et de la forêt dense. 
concerne l'éventail des espèces de noctuidés, la prédominance et la répartition des espèces, la proportion des sexes, les hôtes et les conditions écologiques. De plus, les lépidoptères suspects se sont avérés des espèces ophtalmotropes: Sylopta derogata (Pyralidae), Scopula lubricata (Geometridae).

Cependant, la rareté de leur présence indique qu'ils ne sont que d'occasionnels suceurs de sécrétions lacrymales. Il est également probable que ces suceurs occasionnels se nourrissent aussi d'autres liquides, comme tel est le cas de différentes espèces de géométridés et de pyralidés d'Asie, capables d'inhiber des excrétions et des sécrétions animales (1).

En se basant sur les familles, genres et espèces de lépidoptères ophtalmotropes observés dans les autres régions d'Afrique et dans de nombreux pays d'Asie, il est possible de faire une comparaison préliminaire (tableau VII, p. 328)

Il est évident que la répartition de certains genres ou espèces est considérable en diverses régions d'Asie et d'Afrique. D'autre part, il s'est avéré que certaines espèces concernent des régions assez délimitées, par exemple le cas de Lobocraspis griseifusa : Asie sud-orientale; Enblemma pyrochroa: Afrique occidentale; $E n$ blemma admota: Afrique du sud.

Bien que les recherches de la dernière expédition aient eu lieu à une saison différente de celle de la première, la situation entomologique était globalement la même. On a cependant remarqué une différence: Absence totale des sphingidés Nephele comma et $N$. peneus en automne 1972. Ce fait est regrettable car, de ce fait, on n'a pas pu résoudre le problème du possible ophtalmotropisme de ces deux espèces.

Une autre différence se rapporte aux obser- vations faites sur le ranch d'Abokouamékro. Lors de la seconde expédition, les lépidoptères ophtalmotropes étaient absents. Il nous semble évident que la végétation du ranch est déjà fortement influencée par des éléments de la forêt dense, qui limitent probablement le développement des espèces ophtalmotropes.

En ce qui concerne les conditions écologiques, les recherches ont confirmé les résultats de l'expédition précédente. Cependant, d'après des travaux botaniques plus récents, les limites des zones phytogéographiques ont varié. C'est pourquoi nous avons incorporé à la présente publication une carte montrant les conditions valables à l'époque actuelle. Il s'est avéré de nouveau que les lépidoptères ophtalmotropes sont répartis dans les savanes dites guinéenne et soudanaise, et le Sahel. La forêt dense est donc exclue de cette répartition. Les résultats quant à l'écologie et à la faune serviront à une étude ultérieure, mosaïque constituée par les données élaborées en Asie et en Afrique (16). Cette étude sur les corrélations de la faune et de l'écologie sur la base du bio-climat des différentes régions nécessite cependant des informations plus précises et plus variées.

\section{Remerciements}

Nous exprimons tout particulièrement notre reconnaissance aux Dr. P. LINDLEY, Korhogo, au Dr et à Mme P. VOGEL, Adiopodoumé, M. C. RENWART, Abokouamékro, M. B. PENDRIEZ, Bobo-Dioulasso, et aux nombreux techniciens et assistants africains qui ont contribué au succès de l'expédition. L'identification des Muscidae a été aimablement faite par M. A. C. PONT, Londres, et celle de quelques pyralidés et géométridés par M. D. S. FLETCHER et M. M. SHAFFER, également British Museum (Nat. Hist.), Londres.

\section{SUMMARY}

\section{Complementary observations on ophthalmotropic in west Africa}

During a second expedition to the Yyory Coast, further records of the noctuids Arcyophora patricula, A. longivalvis, $A$. zanderi and $A$. endoglauca have been obtained. During a special excursion to Upper Volta, a first observation of the eye-frequenting behaviour was made in that country. A first record of Arcyophora patricula in Dahomey is mentioned in this paper and the distribution of the various species of Arcyophora in West Africa is given. The eye-frequenting behaviour of Sylepta derogata Fabr. (Pyralidae/Pyranstinae) has been discovered and Scopula lubricata Warr. (Geometridae/Sterrhinae) which was suspected as an ophthalmic species in the previous expedition, has been confirmed. Additional information on the aspects of the distribution, occurrence and 
TABL. NVII Familles, genres et espèces de Iêpidoptëres ophtalmotropes en Afrique et en Asie.

\begin{tabular}{|c|c|c|c|c|}
\hline Familles & Afri que & & A $s$ i e & \\
\hline Noctuidae & $\begin{array}{l}\text { Arcyophora patricula } \\
\text { Arcyophora tongivatuis } \\
\text { Arcyophora zonderi } \\
\text { Arcyophora endogtauca } \\
\text { Arcyophora elegantula } \\
\text { Eubtemma pyrochroa } \\
\text { Eublemma admota }\end{array}$ & $\begin{array}{l}\text { Arcyophora sylvatica } \\
\text { Arcyophora icterica } \\
\text { Aroyophora bothrophora } \\
\text { Lobocraspis griseifusa } \\
\text { Calpe minuticomis (?) }\end{array}$ & & \\
\hline Geometridae & $\begin{array}{l}\text { Semiothisa inaequilinea } \\
\text { Scopula (?) iubricata } \\
\text { Scoputa sp. }\end{array}$ & $\begin{array}{l}\text { Hypochrosis hyadara } \\
\text { Hypochrosis flavifusata } \\
\text { Hypochrosis imis } \\
\text { Hypochrosis pyrrhularia } \\
\text { Hypochrosis sp. } 1 \\
\text { Iypochrosis sp. } 2 \\
\text { Antitrygodes cuneilinea }\end{array}$ & $\begin{array}{l}\text { Scopula attentata } \\
\text { Scopula pulverosa } \\
\text { Scopula fibulata } \\
\text { Somatina anthophilita } \\
\text { Eroblepsis albidior } \\
\text { Eroblepsis conjuntiva } \\
\text { Antitrygodes divisaria }\end{array}$ & $\begin{array}{l}\text { Nobitia turbata } \\
\text { Nobitia obliterata } \\
\text { Semiothisa myandari } \\
\text { Semiothisa nora } \\
\text { Semiothisa elvirata } \\
\text { Semiothisa fasoiala } \\
\text { Semiothisu sp. } 1\end{array}$ \\
\hline Pyratidae & $\begin{array}{l}\text { Filodes cocylusalis } \\
\text { Sylepta sp, près de } \\
\text { fulviteeps } \\
\text { Sylepla derogata } \\
\text { Sylepta balteata } \\
\text { Brandina admixtalis }\end{array}$ & $\begin{array}{l}\text { Botyodes flavibasalis } \\
\text { Botyodes prinaipalis } \\
\text { Meroalena tullatis } \\
\text { Fitodes fulvidursulis } \\
\text { Pionea aureolatis } \\
\text { Pionea damastesalis }\end{array}$ & $\begin{array}{l}\text { Sylepta leopardalis } \\
\text { Sylepta derogata } \\
\text { Syzepta sp. près de } \\
\text { iopardalis } \\
\text { Sylepta sp. } 1 \\
\text { Epipagis pictalis }\end{array}$ & $\begin{array}{l}\text { Pagyda fulvistmigata } \\
\text { Pagyda salvalis } \\
\text { Hamitala sp. } 1 \\
\text { Thliptoceras cascalis }\end{array}$ \\
\hline Notodontidae & & Tarsolepis sommeri & & \\
\hline
\end{tabular}

D'après les informations de Büttiker (Div. publ.) et Bänziger (1972) (Situation fin 1973). 
ecology are given in connection with ophthalmotropic noctuids, pyralids and geometrids. An endoparasitic larval filaria (Mermis sp.) has been isolated from a female of $A$. patricula. The results of further microbiological investigations have been published separately.

\section{RESUMEN}

\section{Observaciones complementarias sobre los lepidópteros oftalmotropos} en Africa del Oeste

Se efectuó una segunda encuesta en 1972 en Costa de Marfil y en Alta Volta para realizar búsquedas complementarias desde el punto de vista entomologico y microbiologico.

Se observaron las mariposas siguientes: Arcyophora patricula Hpsn., A. longiralvis Guen., A. zanderi Feld. e A. endoglauca Hpsn. En Alta Volta, se encontró Arcyophora patricula en los alrededores de BoboDioulasso. Así se pudo confirmar las costumbres oftalmotropas en las especies siguientes: Sylepta derogata Fabr. (Pyralidae) y Scopula lubricata Warr. (Geometridae).

Sin embargo, no se observó ningún ejemplar de Nephele comma (Hopfer) y $N$. peneus (Cramer) en las regiones de Korhogo, Bouake, Abokouamekro y Abidjan. Se hicieron observaciones suplementarias sobre los biotopos; otras publicaciones tratan de la trasmisión de la keratoconjuntivitis a los bovinos.

Se aisló Mermis sp., helminto endoparásito al estado laryario, a partir de una hembra de Arcyophora patricula (Cerca de Bouake).

\section{BIBLIOGRAPHIE}

1. BANZIGER (H.), Biologie der lacriphagen Lepidopteren in Thailand und Malaya. Rev. Suisse Zool., 1972, 79 (4): 1381-2469.

2. BUTTIKER (W.). Biological notes on eye-frequenting moths from N. Thailand. Mitt. Schweiz. ent. Ges., 1966, 39 (3-4) : 151-179.

3. BUTTIKER (W.). First record of eye-frequenting behaviour of Arcyophora patricula Hmps. (Lepid., Noctuidae) in Nigeria. Bull. ent. Soc. Nigeria, 1970, 2: 105-110.

4. BUTTIKER (W.). Observations préliminaires sur les lépidoptères ophtalmotropes dans la République démocratique du Zaïre. Mitt. Schweiz. ent. Ges., 1972, 45 (1-3) : 31-34.

5. BUTTIKER (W.). New observations on eyefrequenting Lepidoptera from Asia. Proc. 13th Intern. Congr. Ent. (Moscow), 1968, 3: 137-138.

6. BÜTTIKER (W.). Vorläufige Beobachtungen an augenbesuchenden Schmetterlingen in der Elfenbeinküste. Rev. Suisse Zool., 1973, 80 (1) : 1.43.

7. BUTTIKER (W.). Observations préliminaires sur les lépidoptères ophtalmotropes dans la République du Mali. Folia parasisologica (Praha), 1973, 20 (3) : 267-271.

8. BUTTIKER (W.), BEZUIDENHOUT (J.D.). First observations of eye-frequenting Lepidoptera in South West Africa, J. ent. Soc. Sthn. Africa 1974, 37 (1) : 73-78.

9. BUTTTKER (W.). Biotopes and their bioclimates of eye-frequenting Lepidoptera (in preparation).

10. CLOUDSLEY-THOMPSON (J. L.). The Zoology of tropical Africa. London, 1969.

11. FLETCHER (D. S.). Communication personnelle, 1966.
12. GUILLAUMET (J. L.), ADJANOHOUN (E.). Le milieu naturel de la Côte d'Ivoire. La végétation. Paris, 1971, pp. 161-263 (et carte de la végétation). (Mémoires ORSTOM no 50 ).

13. JOANNIS (J. de). Observations sur les mours de quelques Noctuidae (Acontinae) et description d'une espèce nouvelle. Bull. Soc. ent. France. Séance du 22 mars 1911: 118-119.

14. KEAY (R.). Vegetation map of Africa. London, Oxford University Press, 1959.

15. NICOLET (J.), BƯTTLKER (W.). Observations sur la kératoconjonctivite infectieuse du bovin en Côte d'Ivoire. I. Aspects microbiologiques. Rev. Elev. Méd. vét. Pays trop., 1975, 28 (2): 115-124.

16. NICOLET (J.), BÜTTIKER (W.). Observations sur la kératoconjonctivite infectieuse du bovin en Côte d'Ivoire. II. Etude sur le rôle vecteur des lépidoptères ophtalmatropes. Rev. Elev. Méd. vét. Pays trop., 1975, 28 (2): 125-132.

17. NICKLE (W.R.). Nematode infections. Textbook of insect pathology (Chapter 7). New York, Dekker, 1972.

18. PLATT (E. E.). List of foodplants of some South African lepidopterous larvae. S. Afr. J. nat. Hist., 1921, 3 : 114.

19. REID (E. T. M.). Observations on feeding habits of adult Arcyophora. Proc. R. ent. Soc. Lond. (B), 1954, 23 (11-12) : 200-204.

20. VIETTE (P.). Communication personnelle, 1970.

21. WELCH (H. E.), POINAR (G. O.). The role and use of Nematodes in the regulation and control of insects of medical importance. Proc. $12 \mathrm{th}$. Intern. Congr. Entomology, London, 1964, p. 732. 